\title{
Security for Two-Way Untrusted Relay against Constant and Reactive Jamming with Fixed Signals
}

\author{
Chan Dai Truyen Thai ${ }^{1}$, Vo Nguyen Quoc Bao ${ }^{2}$, De-Thu Huynh ${ }^{3}$ \\ ${ }^{1}$ Vietnamese-German University (VGU), Vietnam \\ 2 Posts and Telecommunications Institute of Technology (PTIT), Ho Chi Minh City, Vietnam \\ ${ }^{3}$ Ho Chi Minh City University of Economics and Finance (UEF), Vietnam
}

Correspondence: Chan Dai Truyen Thai, chan.ttd@vgu.edu.vn

Communication: received 28 November 2020, revised 31 December 2020, accepted 14 January 2021

Online publication: 16 April 2021, Digital Object Identifier: 10.21553/rev-jec.260

The associate editor coordinating the review of this article and recommending it for publication was Prof. Dang The Ngoc.

\begin{abstract}
Active attacking in physical-layer security has not been significantly studied while potentially causing serious consequences for the legitimate networks. In this paper, we propose a novel method to estimate and remove the jamming signals from multiple multi-antenna jammers in a two-way relay network with multi-antenna legitimate and relay nodes. We carefully consider the signals in the time slots in order to exploit the repetition of the signals and design the transmitted signals which can work in different cases. The numerical results show that the secrecy rate at the legitimate nodes of the proposed scheme is higher than that of the conventional method when considering the affect of transmit signal-to-noise ratio (SNR); the number antennas at the legitimate and relay nodes; normalized distance between one legitimate node and the relay; and the vertical coordinate of the relay.
\end{abstract}

Keywords- Eavesdropper, jamming, physical-layer security, reactive, untrusted relay.

\section{INTRODUCTION}

Physical-layer security has been extensively researched for about two decades. A great number of results and techniques have been created. The view on the malicious agents have also changed, become more diverse, and upgraded over time [1-3]. Generally, the malicious agents can be classified into two main categories: passive and active attacks. The former refers to those nodes only listening to or overhearing signals, trying to extract the most information from them, and using it for malicious purposes including analysis while the later can emit attacking signals.

Eavesdroppers and untrusted relays/cooperators can be considered as passive attackers [1]. The eavesdroppers stay completely in the "dark" and are generally assumed to be unknown by the legitimate nodes in terms of positions and channel gains. In the majority of cases, the distribution of those variables are assumed to be known by the legitimate nodes. The untrusted cooperative nodes will honestly help/cooperate with the legitimate nodes in a way that the secrecy rate is maximized. However, in the meantime, they try to extract as much information as possible from the received/overheard signals and use it for malicious purposes. Other trustworthy levels/trust degrees can also be defined to consider a finer resolution of maliciousness [4].

Even though the active attacking topics has not been extensively researched, there are a number of works in this area. Yan et al. proposed a scheme to remove the jamming signals by variable elimination in equation solving for a point-to-point wireless network however the full description is given for the case of only two antennas and one jammer [5]. Karlsson et al. designed an optimal scheme to jam a pair of single-antenna transmitter and receiver with a direct transmission [6]. In a game-theoretic and multi-antenna scenario in [7], the eavesdropper can choose either eavesdropping and jamming the legitimate nodes in a direct transmission. A few other works considered on jamming attacking in VANETS [8], flying Ad Hoc networks [9], jamming attacking in cognitive radio networks with Stackelberg Game [10].

In an efficient way of using energy, while passive attackers try to maximally receive information, active attackers try to maximally hinder the transmissions of the legitimate [11, 12]. Two examples of actively attacking is jamming and forwarding garbled information [13]. Basically, there are three modes of jamming as follows.

- Constant jamming: the jammers always transmit jamming signals [14].

- Random jamming: the jammers only transmit in random time slots with a certain probability [15].

- Reactive jamming: only when the jammers detect an active transmission from the legitimate nodes, they transmit. However, we assume that due to the delay of the detection, the jammers only start the jamming transmission one time slot after the legitimate nodes start their transmission [16].

In each jamming mode, the jammers can transmit one of two signal types as follows. 
- Fixed signal: each jammer always transmit the same signal.

- Varied signal: each jammer transmit a different signal in every time slot.

In this paper, we propose a novel scheme to estimate and remove the jamming signals in a two-way relay network with multi-antenna legitimate, relaying, and jamming nodes. The two-way relay network is considered because this network model is very popular in practice, e.g., a wireless user is downloading from and uploading to a server [17]. To the best of our knowledge, this is the first work on such a topic. We consider constant and reactive fixed-signal jamming. The numerical results show that the performance of the proposed scheme in both modes is better than that of the conventional scheme.

The rest of the paper is organized as follows. Section 2 describes the system model used in the paper. Section 3 presents the proposed and conventional schemes in the constant jamming mode. From the result of this section, we discuss and deduce the result for the reactive jamming mode, and present in Section 4. The numerical results are presented and analyzed in Section 5 and the conclusion is drawn in Section 6.

\section{System Model}

\subsection{General System Model}

In this part, we describe the general model for both conventional and proposed schemes. A and B, which are referred here to as legitimate nodes, want to send information signal vectors $\mathbf{x}_{\mathrm{A}}$ and $\mathbf{x}_{\mathrm{B}}$, respectively, to each other as shown in Figure 1. However, due to a large distance or a bad faded channel between them, there is not a reliable channel for them to exchange the information and they must rely on the help of relay $R$.

There are $K$ actively attacking nodes which transmit jamming signals to make interference to the transmissions of $\mathrm{A}, \mathrm{B}$, and $\mathrm{R}$. We assume that $\mathrm{A}, \mathrm{B}$, and $\mathrm{R}$ do not have information about all jamming signals and all channels from the jammers to them but know which jamming mode and signal type are used. Nodes A, B, $\mathrm{R}$, and $\mathrm{J}_{k}$ are equipped with $n, n, n_{\mathrm{R}}$, and $n_{\mathrm{J}}$ antennas, respectively. To focus on estimating and cancelling the jamming signals, we assume that the channels between A (B) and R are known among these nodes so that the needed information signals are successfully detected. The channels are fixed in a coherence time of $l$ time slots and change between such periods. Therefore all precoding vectors and matrices can be calculated at A, $\mathrm{B}$, and $\mathrm{R}$.

\subsection{System Model for the Proposed Scheme}

In this part, we describe the system model and common scheme for the proposed scheme in both constant and reactive jamming modes. In order to analyze the characteristics of different scenarios and classify them, we first describe the general transmission scheme which is organized in frames. Each frame consists of

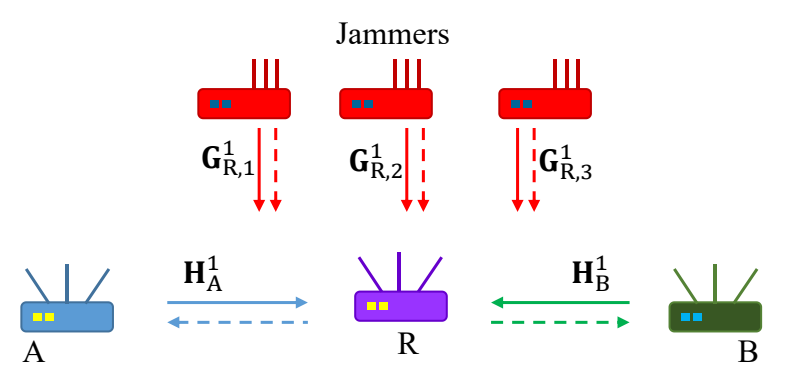

Figure 1. The network model. Transmissions in the first and second frames are represented by solid and dashed arrows, respectively.

two phases. There are $m_{1}$ and $m_{2}$ time slots in the first and second phases, respectively. The designed scheme will depend on the comparison between these numbers and $l$. The channel matrices from $\mathrm{A}$ and $\mathrm{B}$ to $\mathrm{R}$ in slot $i$ of phase $i_{\mathrm{P}}$ are denoted by $\mathbf{H}_{\mathrm{A}}^{i_{\mathrm{P}}, i}$ and $\mathbf{H}_{\mathrm{B}}^{i_{\mathrm{P}}, i}$, respectively.

The channel matrices from the $k$-th jammer to $\mathrm{A}, \mathrm{B}$, and $\mathrm{R}$ in slot $i$ of phase $i_{\mathrm{P}}$ are denoted by $\mathrm{G}_{\mathrm{A}, k^{\prime}}^{i_{\mathrm{P}}, i} \mathrm{G}_{\mathrm{B}, k^{\prime}}^{i_{\mathrm{P}}, i}$ and $\mathrm{G}_{\mathrm{R}, k^{\prime}}^{i_{\mathrm{P}}, i}$ respectively. The noise in time slot $i$ of phase $i_{\mathrm{P}} \in\{1,2\}$ at node $i_{\mathrm{N}} \in\{\mathrm{R}, \mathrm{B}\}$ is denoted by $z_{i_{\mathrm{N}}, i}^{i_{\mathrm{p}}}$. We denote $1 \times n$ vectors with all entries of 1 and 0 by $\mathbf{1}_{n}$ and $\mathbf{0}_{n}$, respectively; element $i$ and elements $i_{1}$ to $i_{2}$ of vector a by $[\mathbf{a}]_{i}$ and a by $[\mathbf{a}]_{i_{1}}^{i_{2}}$, respectively; element $(i, j)$, column $j$, and row $i$ of matrix $\mathbf{A}$ by $[\mathbf{A}]_{i, j},[\mathbf{A}]_{(:, j)}$ and $[\mathbf{A}]_{(i,:)}$, respectively; and the determinant of matrix $\mathbf{A}$ by $|\mathbf{A}|=\operatorname{det}(\mathbf{A})$. Assume we can write $\mathbf{A}=\left\{[\mathbf{A}]_{i, j}\right\}$.

In slot $i$ of phase $1, \mathrm{~A}$ and $\mathrm{B}$ transmit the $i$-th elements of $\mathbf{x}_{\mathrm{A}}$ and $\mathrm{x}_{\mathrm{B}}$, respectively. The received signal at $\mathrm{R}$ is given by

$$
\mathbf{y}_{\mathrm{R}}^{1, i}=\mathbf{H}_{\mathrm{A}}^{1, i} \mathbf{u}_{\mathrm{A}} x_{\mathrm{A}}^{i}+\mathbf{H}_{\mathrm{B}}^{1, i} \mathbf{u}_{\mathrm{B}} x_{\mathrm{B}}^{i}+\sum_{k=1}^{K} \mathbf{G}_{\mathrm{R}, k}^{1, i} \mathbf{x}_{\mathrm{J}, k}^{1, i}+\mathbf{z}_{\mathrm{R}}^{1, i}
$$

where $1 \leq i \leq m_{1} ; u_{\mathrm{A}}$ and $u_{\mathrm{A}}$ are corresponding precoding vectors. $\mathbf{H}_{\mathrm{A}}^{1, i} \mathbf{u}_{\mathrm{A}} x_{\mathrm{A}}^{i}+\mathbf{H}_{\mathrm{B}}^{1, i} \mathbf{u}_{\mathrm{B}} x_{\mathrm{B}}^{i}$ is referred to as the mixed information signal. In slot $j$ of phase $2, R$ precodes $\mathbf{y}_{\mathrm{R}}^{1, i}$ with matrix $\mathbf{B}_{\mathrm{B}}^{j i} \in \mathcal{C}^{n_{\mathrm{R}} \times n_{\mathrm{R}}}$ and transmit it to $A$ and $B$. The received signal at $B$ is given by

$$
\begin{aligned}
\mathbf{y}_{\mathrm{B}}^{2, j}= & \mathbf{H}_{\mathrm{B}}^{2, j^{+}} \sum_{i=1}^{m_{1}} \mathbf{B}_{\mathrm{B}}^{j i} \mathbf{y}_{\mathrm{R}}^{1, i}+\sum_{k=1}^{K} \mathbf{G}_{\mathrm{B}, k}^{2, j} \mathbf{x}_{\mathrm{J}, k}^{2, j}+\mathbf{z}_{\mathrm{B}}^{2, j} \\
= & \mathbf{H}_{\mathrm{B}}^{2, j^{+}} \sum_{i=1}^{m_{1}} \mathbf{B}_{\mathrm{B}}^{j i} \mathbf{H}_{\mathrm{A}}^{1, i} \mathbf{u}_{\mathrm{A}} x_{\mathrm{A}}^{i}+\mathbf{H}_{\mathrm{B}}^{2, j^{\dagger}} \sum_{i=1}^{m_{1}} \mathbf{B}_{\mathrm{B}}^{j i} \mathbf{H}_{\mathrm{B}}^{1, i} \mathbf{u}_{\mathrm{B}} x_{\mathrm{B}}^{i} \\
& +\mathbf{H}_{\mathrm{B}}^{2, j} \sum_{i=1}^{m_{1}} \mathbf{B}_{\mathrm{B}}^{j i} \sum_{k=1}^{K} \mathbf{G}_{\mathrm{R}, k}^{1, i} \mathbf{x}_{\mathrm{J}, k}^{1, i} \\
& +\mathbf{H}_{\mathrm{B}}^{2, j} \sum_{i=1}^{m_{1}} \mathbf{B}_{\mathrm{B}}^{j i} \mathbf{z}_{\mathrm{R}}^{1, i}+\sum_{k=1}^{K} \mathbf{G}_{\mathrm{B}, k}^{2, j} \mathbf{x}_{\mathrm{J}, k}^{2, j}+\mathbf{z}_{\mathrm{B}}^{2, j}
\end{aligned}
$$

where $1 \leq j \leq m_{2}$. Since $\mathbf{x}_{\mathrm{B}}^{i}$, channels, precoding vectors and matrices are available at $\mathrm{B}$, it can remove the second term in $\mathbf{y}_{\mathrm{B}}^{2, j}$. The achieved signal then given by

$$
\begin{aligned}
\tilde{\mathbf{y}}_{\mathrm{B}}^{2, j}= & \mathbf{H}_{\mathrm{B}}^{2, j^{\dagger}} \sum_{i=1}^{m_{1}} \mathbf{B}_{\mathrm{B}}^{j i} \mathbf{H}_{\mathrm{A}}^{1, i} \mathbf{u}_{\mathrm{A}} x_{\mathrm{A}}^{i}+\mathbf{H}_{\mathrm{B}}^{2, j^{\dagger}} \sum_{i=1}^{m_{1}} \mathbf{B}_{\mathrm{B}}^{j i} \mathbf{g}_{\mathrm{R}}^{1, i} \\
& +\mathbf{H}_{\mathrm{B}}^{2, j^{\dagger}} \sum_{i=1}^{m_{1}} \mathbf{B}_{\mathrm{B}}^{j i} \mathbf{z}_{\mathrm{R}}^{1, i}+\mathbf{g}_{\mathrm{B}}^{2, j}+\mathbf{z}_{\mathrm{B}}^{2, j}
\end{aligned}
$$


where $\mathbf{g}_{\mathrm{R}}^{1, i}=\sum_{k=1}^{K} \mathbf{G}_{\mathrm{R}, k}^{1, i} \mathbf{x}_{\mathrm{J}, k^{\prime}}^{1, i}$ and $\mathbf{g}_{\mathrm{B}}^{2, j}=\sum_{k=1}^{K} \mathbf{G}_{\mathrm{B}, k}^{2, j} \mathbf{x}_{\mathrm{J}, k}^{2, j}$. We refer $\mathbf{g}_{\mathrm{R}}^{1, i}$ and $\mathbf{g}_{\mathrm{B}}^{2, j}$ to as jamming components of the first and second phases, respectively; and $\mathrm{G}_{\mathrm{R}, k^{\prime}}^{1, i} \mathrm{G}_{\mathrm{B}, k^{\prime}}^{2, j} \mathbf{x}_{\mathrm{J}, k^{\prime}}^{1, i}$ $\mathbf{x}_{\mathrm{J}, k}^{2, j}$ as jamming factors. Generally, there are methods to decode needed information signal $x_{\mathrm{A}}^{i}$ as follows.

- B decodes $x_{\mathrm{A}}^{i}$ treating all jamming components as noise. This method gives a low performance, especially when the jamming powers are high or the channels from the jammers to B are good.

- B estimates all jamming components first, cancels them in $\tilde{\mathbf{y}}_{\mathrm{B}}^{2, j}$, and decodes $x_{\mathrm{A}}^{i}$. This is impossible since $\mathrm{B}$ does not achieve enough signals to decode the needed signals. To demonstrate this, we consider two cases as follows in the most general scenario in which all channels and jamming signals change every time slot.

- Estimating each jamming component in factors: B first needs to estimate all $\left(m_{1}+m_{2}\right) K n_{\text {J }}$ jamming signals $\left(\mathbf{x}_{\mathrm{J}, k^{\prime}}^{1, i} \mathbf{x}_{\mathrm{J}, k}^{2, j}\right)$ in two phases; all $m_{1} K n_{\mathrm{J}} n_{\mathrm{R}}$ channels from the jammers to $\mathrm{R}$ in phase 1 ; all $m_{2} K n_{\mathrm{J}} n$ channels from the jammers to $\mathrm{B}$ in phase 2 . However, $\mathrm{B}$ achieves only $m_{2} n$ signals in vector $\tilde{\mathbf{y}}_{\mathrm{B}}^{2, j}$ while $m_{2} n$ is much smaller than $\left(m_{1}+m_{2}\right) K n_{\mathrm{J}}+m_{1} K n_{\mathrm{J}} n_{\mathrm{R}}+m_{2} K n_{\mathrm{J}} n$.

- Estimating each jamming component as a whole: we reduce the number of variables to be estimated by only estimating each component as a whole $\left(\mathbf{g}_{\mathrm{R}}^{1, i}\right.$ or $\left.\mathbf{g}_{\mathrm{B}}^{2, j}\right)$ and does not need to estimate each factor inside $\left(\mathbf{G}_{\mathrm{R}, k^{\prime}}^{1, i} \mathbf{G}_{\mathrm{B}, k^{\prime}}^{2, j} \mathbf{x}_{\mathrm{J}, k^{\prime}}^{1, i} \mathbf{x}_{\mathrm{J}, k}^{2, j}\right)$. In this case, $\mathrm{B}$ has $m_{1} n_{\mathrm{R}}+m_{2} n+m_{1}$ signals to decode, including $m_{1} n_{\mathrm{R}}$ signals in all $\mathbf{g}_{\mathrm{R}}^{1, i}$, $m_{2} n$ signals in all $\mathbf{g}_{\mathrm{B}}^{2, i}$, and $m_{1}$ signals in $\mathbf{x}_{\mathrm{A}}$. However, again $m_{2} n$ is also much smaller than $m_{1} n_{\mathrm{R}}+m_{2} n+m_{1}$.

- We design the transmission scheme and the precoding matrices so as in phase $1, \mathrm{R}$ can estimate each jamming component as a whole or in factors, cancel them, amplify and forward a jamming-free version of the mixed information signal to $\mathrm{A}$ and $\mathrm{B}$ in phase 2; and in phase 2, A and B can estimate each jamming component as a whole or in factors, cancel them, and decode the information signal. In this paper, we use this third method and explain about it in more details below.

The way we design the scheme depends on which jamming mode and jamming signal type are used as well as how long the coherence time is. In fact, jamming components $\mathbf{g}_{\mathrm{R}}^{1, i}$ and $\mathbf{g}_{\mathrm{B}}^{2, j}$ depend on the channels $\left(\mathbf{G}_{\mathrm{R}, k}^{1, i}, \mathrm{G}_{\mathrm{R}, k}^{2, i}\right)$ and jamming signals $\left(\mathbf{x}_{\mathrm{J}, k}^{1, i}, \mathbf{x}_{\mathrm{J}, k}^{2, j}\right)$. The channels are decided by the surrounding environment while the jamming signals are decided by the jammers. Jamming signals may vary slower than the channels with a short-enough coherence time and, e.g., constant jamming strategy with fixed signals. On the other hand, jamming signals may vary faster than the channels with a long-enough coherence time and, e.g., reactive jamming strategy with varied signals. The varying rate of the jamming components is the rate of the one, of the two factors, channels and signals, which varies faster. In addition, the faster the factors vary, the more difficult it is for us to estimate the jamming components. In this paper, we consider all three jamming modes with both signal types and coherence time $l \geq 2$ since with $l=1$, the jamming components vary the fastest and there is not enough information to estimate them. Furthermore, $l \geq 2$ is reasonable and usually assumed for wireless cooperative/relaying networks.

The jamming signal from each jammer is always the same and that from the $k$-th jammer is given by

$$
\mathbf{x}_{\mathrm{J}, k}^{1, i}=\mathbf{x}_{\mathrm{J}, k}^{2, j}= \begin{cases}\mathbf{a}_{\mathrm{J}, k}, & \text { when jamming, } \\ \mathbf{0}_{n_{\mathrm{J}}}, & \text { when not jamming. }\end{cases}
$$

In case all jammers transmit with the same signal, $\mathbf{a}_{\mathrm{J}, k}=\mathbf{a}_{\mathrm{j}}, \forall k$. However, as we will try to decode and cancel jamming component $\mathbf{g}_{\mathrm{R}}^{1, i}$ and $\mathbf{g}_{\mathrm{B}}^{2, i}$ as a whole, not the individual jamming signals from jammers, that the jamming signals from different jammers are the same or not is not important. However, we assume that in a time slot all jammers transmit jamming signals or all jammers do not transmit jamming signals. The case in which some jammers transmit while others do not in a time slot is not considered in this section. To improve the average rate, we design that the coherence time fits into each phase, i.e., each of the two phases consists of $l$ time slots. In this section, we consider only two cases: all jammers transmit in a time slot with probability $p_{\mathrm{J}}$; and no jammer transmits, with probability $1-p_{\mathrm{J}}$.

\section{Constant Jamming}

\subsection{Conventional Scheme}

In this scheme, we assume that all jamming signals cannot be estimated and removed, therefore R, A, and $B$ will treat them as noise. In return, they use all time slots for transmitting information signals instead of sacrificing one for estimating the jamming components. The scheme is performed every two time slots and does not depend on the coherence time. The noise vectors at node $R$ and $B$ are respectively denoted by $z_{R}$ and $z_{B}$ below. In the first slot, the received signal at $R$ is given by

$$
\mathbf{y}_{\mathrm{R}}=\mathbf{H}_{\mathrm{A}}^{1} \mathbf{u}_{\mathrm{A}} x_{\mathrm{A}}+\mathbf{H}_{\mathrm{B}}^{1} \mathbf{u}_{\mathrm{B}} x_{\mathrm{B}}+\mathbf{g}_{\mathrm{R}}+\mathbf{z}_{\mathrm{R}} .
$$

The transmitted signal by $R$ in the second slot is given by

$$
\begin{aligned}
\mathbf{x}_{\mathrm{R}} & =\frac{1}{\sqrt{\alpha}} \mathbf{y}_{\mathrm{R}} \\
& =\frac{1}{\sqrt{\alpha}}\left(\mathbf{H}_{\mathrm{A}}^{1} \mathbf{u}_{\mathrm{A}} x_{\mathrm{A}}+\mathbf{H}_{\mathrm{B}}^{1} \mathbf{u}_{\mathrm{B}} x_{\mathrm{B}}+\mathbf{g}_{\mathrm{R}}+\mathbf{z}_{\mathrm{R}}\right)
\end{aligned}
$$

where

$$
\begin{gathered}
\alpha=\mathbb{E}\left[\mathbf{y}_{\mathrm{R}}^{\dagger} \mathbf{y}_{\mathrm{R}}\right]=\mathbf{u}_{\mathrm{A}}^{\dagger} \mathbf{H}_{\mathrm{A}}^{1^{\dagger}} \mathbf{H}_{\mathrm{A}}^{1} \mathbf{u}_{\mathrm{A}}+\mathbf{u}_{\mathrm{B}}^{\dagger} \mathbf{H}_{\mathrm{B}}^{1^{\dagger}} \mathbf{H}_{\mathrm{B}}^{1} \mathbf{u}_{\mathrm{B}} \\
+\sum_{k=1}^{K} p_{\mathrm{J}, k} \operatorname{tr}\left\{\mathbf{G}_{\mathrm{R}, k}^{\dagger} \mathbf{G}_{\mathrm{R}, k}\right\}+n_{\mathrm{R}} \sigma^{2} .
\end{gathered}
$$


The received signal at $B$ is given by

$$
\begin{aligned}
\mathbf{y}_{\mathrm{B}}= & \frac{1}{\sqrt{\alpha}} \mathbf{H}_{\mathrm{B}}^{2^{\dagger}}\left(\mathbf{H}_{\mathrm{A}}^{1} \mathbf{u}_{\mathrm{A}} x_{\mathrm{A}}+\mathbf{H}_{\mathrm{B}}^{1} \mathbf{u}_{\mathrm{B}} x_{\mathrm{B}}+\mathbf{g}_{\mathrm{R}}+\mathbf{z}_{\mathrm{R}}\right) \\
& +\sum_{k=1}^{K} \mathbf{G}_{\mathrm{B}, k} \mathbf{a}_{\mathrm{J}, k}+\mathbf{z}_{\mathrm{B}} .
\end{aligned}
$$

After the known component is removed, the signal is given by

$$
\begin{aligned}
\tilde{\mathbf{y}}_{\mathrm{B}}= & \frac{1}{\sqrt{\alpha}} \mathbf{H}_{\mathrm{B}}^{2^{+}}\left(\mathbf{H}_{\mathrm{A}}^{1} \mathbf{u}_{\mathrm{A}} x_{\mathrm{A}}+\mathbf{g}_{\mathrm{R}}+\mathbf{z}_{\mathrm{R}}^{1}\right) \\
& +\sum_{k=1}^{K} \mathbf{G}_{\mathrm{B}, k} \mathbf{a}_{\mathrm{J}, k}+\mathbf{z}_{\mathrm{B}} .
\end{aligned}
$$

The MAR at B is given by (10) at the beginning of the next page where subscript " $C$ " refer to the conventional scheme. Similarly, the MAR at A is given by (11) at the beginning of the next page. $\mathrm{R}$ estimates $x_{\mathrm{A}}^{i}$ and $x_{\mathrm{B}}^{i}$ with MARs respectively given by (12) and (13) at the beginning of the next page. The secrecy MARS is then given by

$$
r_{\mathrm{C}-\mathrm{Co}}=\left(r_{\mathrm{C}-\mathrm{Co}}^{\mathrm{A}}-r_{\mathrm{C}-\mathrm{Co}}^{\mathrm{A}-\mathrm{R}}\right)^{+}+\left(r_{\mathrm{C}-\mathrm{Co}}^{\mathrm{B}}-r_{\mathrm{C}-\mathrm{Co}}^{\mathrm{B}-\mathrm{R}}\right)^{+} .
$$

\subsection{Proposed Scheme}

The jammers always jam with the same signals therefore, we design such that in first time slot of each phase of the first frame, the transmitter does not transmit any signal. The intended receiver thus receives only the jamming signals and noise. It needs to estimate the jamming signals and use them to cancel their contribution in the received signals in the next time slots. Since the channels are fixed in each phase, we replace superscripts $i$ and $j$ of $\mathbf{H}$ and $\mathbf{u}$ by 1 and 2, respectively.

In the first time slot of the first phase, A and B transmit no signal so the signal received at $R$ is given by

$$
\mathbf{y}_{\mathrm{R}}^{1}=\mathbf{g}_{\mathrm{R}}+\mathbf{z}_{\mathrm{R}}^{1}
$$

where $\mathbf{g}_{\mathrm{R}} \triangleq \sum_{k=1}^{K} \mathbf{G}_{\mathrm{R}, k}^{1} \mathbf{a}_{\mathrm{J}, k}$. For simplicity, in this paper we use Zero Forcing to estimate the jamming component as $\hat{\mathrm{g}}_{\mathrm{R}}^{1}=\mathbf{y}_{\mathrm{R}}^{1}{ }^{*}$ In the $i$-th time slot of the first phase, $2 \leq i \leq l$, $\mathrm{A}$ and $\mathrm{B}$ transmit their respective information signals so the signal received at $\mathrm{R}$ is given by

$$
\mathbf{y}_{\mathrm{R}}^{i}=\mathbf{H}_{\mathrm{A}}^{1} \mathbf{u}_{\mathrm{A}} x_{\mathrm{A}}^{i}+\mathbf{H}_{\mathrm{B}}^{1} \mathbf{u}_{\mathrm{B}} x_{\mathrm{B}}^{i}+\mathbf{g}_{\mathrm{R}}+\mathbf{z}_{\mathrm{R}}^{i}
$$

where $2 \leq i \leq l$. In the first time slot of the second phase, $R$ does not transmit any signals so that $B$ receives only the jamming signals and noise given by

$$
\mathbf{y}_{\mathrm{B}}^{1}=\sum_{k=1}^{K} \mathbf{G}_{\mathrm{B}, k}^{2} \mathbf{a}_{\mathrm{J}, k}+\mathbf{z}_{\mathrm{B}}^{1} \text {. }
$$

In the $j$-th time slot of the second phase, $\mathrm{R}$ calculates

$$
\begin{aligned}
\tilde{\mathbf{y}}_{\mathrm{R}}^{i} & =\mathbf{y}_{\mathrm{R}}^{i}-\mathbf{g}_{\mathrm{R}}=\mathbf{y}_{\mathrm{R}}^{1, i}-\mathbf{y}_{\mathrm{R}}^{1} \\
& =\mathbf{H}_{\mathrm{A}}^{1} \mathbf{u}_{\mathrm{A}} x_{\mathrm{A}}^{i}+\mathbf{H}_{\mathrm{B}}^{1} \mathbf{u}_{\mathrm{B}} x_{\mathrm{B}}^{i}+\mathbf{z}_{\mathrm{R}}^{i}-\mathbf{z}_{\mathrm{R}^{\prime}}^{1}
\end{aligned}
$$

*If MMSE is used, $\hat{\mathrm{g}}_{\mathrm{R}}^{1}=\Lambda_{\mathrm{R}} \mathbf{y}_{\mathrm{R}}^{1}$ where $\Lambda_{\mathrm{R}}$ is a diagonal matrix in which element $(i, i)$ is given by $\Lambda_{\mathrm{R}}[i, i]=\frac{\sum_{k=1}^{K}\left|\left[\mathbf{G}_{\mathrm{R}, k}^{1}\right]_{i}\right|^{2}\left[\mathbf{p}_{\mathrm{J}, k}\right]_{i}}{\sum_{k=1}^{K}\left|\left[\mathbf{G}_{\mathrm{R}, k}^{1}\right]_{i}\right|^{2}\left[\mathbf{p}_{\mathrm{J}, k}\right]_{i}+\sigma^{2}}$. where $2 \leq j \leq l$, to remove $\mathbf{g}_{\mathrm{R}}^{1}$ and transmits $\mathbf{x}_{\mathrm{R}}^{j}$, which is free of jamming signals and given by

$$
\begin{aligned}
\mathbf{x}_{\mathrm{R}}^{j} & =\frac{1}{\sqrt{\alpha}} \tilde{\mathbf{y}}_{\mathrm{R}}^{i} \\
& =\frac{1}{\sqrt{\alpha}}\left(\mathbf{H}_{\mathrm{A}}^{1} \mathbf{u}_{\mathrm{A}} x_{\mathrm{A}}^{i}+\mathbf{H}_{\mathrm{B}}^{1} \mathbf{u}_{\mathrm{B}} x_{\mathrm{B}}^{i}+\mathbf{z}_{\mathrm{R}}^{i}-\mathbf{z}_{\mathrm{R}}^{1}\right)
\end{aligned}
$$

where

$$
\begin{aligned}
\alpha & =\mathbb{E}\left[\mathbf{y}_{\mathrm{R}}^{\dagger} \mathbf{y}_{\mathrm{R}}\right] \\
& =\mathbf{u}_{\mathrm{A}}^{\dagger} \mathbf{H}_{\mathrm{A}}^{1^{\dagger}} \mathbf{H}_{\mathrm{A}}^{1} \mathbf{u}_{\mathrm{A}}+\mathbf{u}_{\mathrm{B}}^{\dagger} \mathbf{H}_{\mathrm{B}}^{1^{\dagger}} \mathbf{H}_{\mathrm{B}}^{1} \mathbf{u}_{\mathrm{B}}+2 n_{\mathrm{R}} \sigma^{2} .
\end{aligned}
$$

In section 2.2, we have used precoding matrix $\mathbf{B}_{\mathrm{B}}^{j i}$ for the transmitted signal from $\mathrm{R}$. This can be used for a general view of the readers. However, optimizing this matrix can lead to very complicated content which may require a lot of other works. Therefore, in this paper we assume $\mathbf{B}_{\mathrm{B}}^{j i}=\mathbf{I}_{n_{\mathrm{R}}}$ which is a $n_{\mathrm{R}} \times n_{\mathrm{R}}$ eye matrix. The received signal at $B$ is given by

$$
\begin{aligned}
\mathbf{y}_{\mathrm{B}}^{j}= & \frac{1}{\sqrt{\alpha}} \mathbf{H}_{\mathrm{B}}^{2^{\dagger}}\left(\mathbf{H}_{\mathrm{A}}^{1} \mathbf{u}_{\mathrm{A}} x_{\mathrm{A}}^{i}+\mathbf{H}_{\mathrm{B}}^{1} \mathbf{u}_{\mathrm{B}} x_{\mathrm{B}}^{i}+\mathbf{z}_{\mathrm{R}}^{1, i}-\mathbf{z}_{\mathrm{R}}^{1,1}\right) \\
& +\sum_{k=1}^{K} \mathbf{G}_{\mathrm{B}, k}^{2} \mathbf{a}_{\mathrm{J}, k}+\mathbf{z}_{\mathrm{B}}^{j} .
\end{aligned}
$$

$B$ also uses similar technique used by $R$ in (18) to remove the jamming component by calculating

$$
\begin{aligned}
\mathbf{y}_{\mathrm{B}}^{j}-\mathbf{y}_{\mathrm{B}}^{1}= & \frac{1}{\sqrt{\alpha}} \mathbf{H}_{\mathrm{B}}^{2^{\dagger}}\left(\mathbf{H}_{\mathrm{A}}^{1} \mathbf{u}_{\mathrm{A}} x_{\mathrm{A}}^{i}+\mathbf{H}_{\mathrm{B}}^{1} \mathbf{u}_{\mathrm{B}} x_{\mathrm{B}}^{i}\right. \\
& \left.+\mathbf{z}_{\mathrm{R}}^{1, i}-\mathbf{z}_{\mathrm{R}}^{1,1}\right)+\mathbf{z}_{\mathrm{B}}^{j}-\mathbf{z}_{\mathrm{B}}^{1} .
\end{aligned}
$$

Since $\mathrm{H}_{\mathrm{B}}^{1}, \mathbf{u}_{\mathrm{B}}$, and $x_{\mathrm{B}}^{i}$ are known at $\mathrm{B}$, it removes the second term in (22) and gets

$$
\tilde{\mathbf{y}}_{\mathrm{B}}=\frac{1}{\sqrt{\alpha}} \mathbf{H}_{\mathrm{B}}^{2^{\dagger}}\left(\mathbf{H}_{\mathrm{A}}^{1} \mathbf{u}_{\mathrm{A}} x_{\mathrm{A}}^{i}+\mathbf{z}_{\mathrm{R}}^{1, i}-\mathbf{z}_{\mathrm{R}}^{1,1}\right)+\mathbf{z}_{\mathrm{B}}^{j}-\mathbf{z}_{\mathrm{B}}^{1} .
$$

Since there two phases each with $l$ time slots and the first time slot in each phase is used to calibrate the jamming component, the scheme can only transfer $l-1$. messages from $\mathrm{A}$ to $\mathrm{B}$. The maximum achievable rate (MAR) at $B$ is given by

$$
r_{\mathrm{P}-\mathrm{Co}}^{\mathrm{B}}=\frac{l-1}{2 l} \log _{2}\left(1+\frac{\mathbf{u}_{\mathrm{A}}^{\dagger} \mathbf{H}_{\mathrm{A}}^{1^{\dagger}} \mathbf{H}_{\mathrm{B}}^{2} \mathbf{H}_{\mathrm{B}}^{2^{\dagger}} \mathbf{H}_{\mathrm{A}}^{1} \mathbf{u}_{\mathrm{A}}}{2 \sigma^{2} \operatorname{tr}\left\{\mathbf{H}_{\mathrm{B}}^{2} \mathbf{H}_{\mathrm{B}}^{2^{\dagger}}\right\}+2 \alpha n \sigma^{2}}\right)
$$

where subscripts " $\mathrm{P}$ " and "Co" refer to the proposed scheme and constant jamming, respectively. Similarly, A can remove the jamming component and known term [18]. The MAR at A is therefore given by

$$
r_{\mathrm{P}-\mathrm{Co}}^{\mathrm{A}}=\frac{l-1}{2 l} \log _{2}\left(1+\frac{\mathbf{u}_{\mathrm{B}}^{\dagger} \mathbf{H}_{\mathrm{B}}^{1^{\dagger}} \mathbf{H}_{\mathrm{A}}^{2} \mathbf{H}_{\mathrm{A}}^{2^{\dagger}} \mathbf{H}_{\mathrm{B}}^{1} \mathbf{u}_{\mathrm{B}}}{2 \sigma^{2} \operatorname{tr}\left\{\mathbf{H}_{\mathrm{A}}^{2} \mathbf{H}_{\mathrm{A}}^{2^{\dagger}}\right\}+2 \alpha n \sigma^{2}}\right) .
$$

$\mathrm{R}$ estimates $x_{\mathrm{A}}^{i}$ and $x_{\mathrm{B}}^{i}$ with the MARs respectively given by

$$
r_{\mathrm{P}-\mathrm{Co}}^{\mathrm{B}-\mathrm{R}}=\frac{l-1}{2 l} \log _{2}\left(1+\frac{\mathbf{u}_{\mathrm{A}}^{\dagger} \mathbf{H}_{\mathrm{A}}^{+} \mathbf{H}_{\mathrm{A}}^{1} \mathbf{u}_{\mathrm{A}}}{\mathbf{u}_{\mathrm{B}}^{\dagger} \mathbf{H}_{\mathrm{B}}^{1^{\dagger}} \mathbf{H}_{\mathrm{B}}^{1} \mathbf{u}_{\mathrm{B}}+2 \sigma^{2}}\right),
$$




$$
\begin{aligned}
& r_{\mathrm{C}-\mathrm{Co}}^{\mathrm{B}}=\frac{1}{2} \log _{2}\left(1+\frac{\mathbf{u}_{\mathrm{A}}^{\dagger} \mathbf{H}_{\mathrm{A}}^{1^{\dagger}} \mathbf{H}_{\mathrm{B}}^{2} \mathbf{H}_{\mathrm{B}}^{2^{+}} \mathbf{H}_{\mathrm{A}}^{1} \mathbf{u}_{\mathrm{A}}}{\sum_{k=1}^{K} p_{\mathrm{J}, k} \operatorname{tr}\left\{\mathbf{G}_{\mathrm{R}, k}^{+} \mathbf{H}_{\mathrm{B}}^{2} \mathbf{H}_{\mathrm{B}}^{2^{\dagger}} \mathbf{G}_{\mathrm{R}, k}\right\}+\sigma^{2} \operatorname{tr}\left\{\mathbf{H}_{\mathrm{B}}^{2} \mathbf{H}_{\mathrm{B}}^{2^{+}}\right\}+\alpha \sum_{k=1}^{K} p_{\mathrm{J}, k} \operatorname{tr}\left\{\mathbf{G}_{\mathrm{B}, k}^{+} \mathbf{G}_{\mathrm{B}, k}\right\}+\alpha n \sigma^{2}}\right) . \\
& r_{\mathrm{C}-\mathrm{Co}}^{\mathrm{A}}=\frac{1}{2} \log _{2}\left(1+\frac{\mathbf{u}_{\mathrm{B}}^{\dagger} \mathbf{H}_{\mathrm{B}}^{+} \mathbf{H}_{\mathrm{A}}^{2} \mathbf{H}_{\mathrm{A}}^{2^{+}} \mathbf{H}_{\mathrm{B}}^{1} \mathbf{u}_{\mathrm{B}}}{\sum_{k=1}^{K} p_{\mathrm{J}, k} \operatorname{tr}\left\{\mathbf{G}_{\mathrm{R}, k}^{+} \mathbf{H}_{\mathrm{A}}^{2} \mathbf{H}_{\mathrm{A}}^{2^{\dagger}} \mathbf{G}_{\mathrm{R}, k}\right\}+\sigma^{2} \operatorname{tr}\left\{\mathbf{H}_{\mathrm{A}}^{2} \mathbf{H}_{\mathrm{A}}^{2^{+}}\right\}+\alpha \sum_{k=1}^{K} p_{\mathrm{J}, k} \operatorname{tr}\left\{\mathbf{G}_{\mathrm{A}, k}^{+} \mathbf{G}_{\mathrm{A}, k}\right\}+\alpha n \sigma^{2}}\right) . \\
& r_{\mathrm{C}-\mathrm{Co}}^{\mathrm{B}-\mathrm{R}}=\frac{1}{2} \log _{2}\left(1+\frac{\mathbf{u}_{\mathrm{A}}^{\dagger} \mathbf{H}_{\mathrm{A}}^{1^{\dagger}} \mathbf{H}_{\mathrm{A}}^{1} \mathbf{u}_{\mathrm{A}}}{\mathbf{u}_{\mathrm{B}}^{\dagger} \mathbf{H}_{\mathrm{B}}^{1^{\dagger}} \mathbf{H}_{\mathrm{B}}^{1} \mathbf{u}_{\mathrm{B}}+\sum_{k=1}^{K} p_{\mathrm{J}, k} \operatorname{tr}\left\{\mathbf{G}_{\mathrm{R}, k}^{+} \mathbf{G}_{\mathrm{R}, k}\right\}+n_{\mathrm{R}} \sigma^{2}}\right), \\
& r_{\mathrm{C}-\mathrm{Co}}^{\mathrm{A}-\mathrm{R}}=\frac{1}{2} \log _{2}\left(1+\frac{\mathbf{u}_{\mathrm{B}}^{\dagger} \mathbf{H}_{\mathrm{B}}^{+} \mathbf{H}_{\mathrm{B}}^{1} \mathbf{u}_{\mathrm{B}}}{\mathbf{u}_{\mathrm{A}}^{\dagger} \mathbf{H}_{\mathrm{A}}^{1^{\dagger}} \mathbf{H}_{\mathrm{A}}^{1} \mathbf{u}_{\mathrm{A}}+\sum_{k=1}^{K} p_{\mathrm{J}, k} \operatorname{tr}\left\{\mathbf{G}_{\mathrm{R}, k}^{+} \mathbf{G}_{\mathrm{R}, k}\right\}+n_{\mathrm{R}} \sigma^{2}}\right) .
\end{aligned}
$$

$$
r_{\mathrm{P}-\mathrm{Co}}^{\mathrm{A}-\mathrm{R}}=\frac{l-1}{2 l} \log _{2}\left(1+\frac{\mathbf{u}_{\mathrm{B}}^{\dagger} \mathbf{H}_{\mathrm{B}}^{1^{\dagger}} \mathbf{H}_{\mathrm{B}}^{1} \mathbf{u}_{\mathrm{B}}}{\mathbf{u}_{\mathrm{A}}^{\dagger} \mathbf{H}_{\mathrm{A}}^{1^{\dagger}} \mathbf{H}_{\mathrm{A}}^{1} \mathbf{u}_{\mathrm{A}}+2 \sigma^{2}}\right) .
$$

The secrecy rate is therefore given by

$$
r_{\mathrm{P}-\mathrm{Co}}=\left(r_{\mathrm{P}-\mathrm{Co}}^{\mathrm{A}}-r_{\mathrm{P}-\mathrm{Co}}^{\mathrm{A}-\mathrm{R}}\right)^{+}+\left(r_{\mathrm{P}-\mathrm{Co}}^{\mathrm{B}}-r_{\mathrm{P}-\mathrm{Co}}^{\mathrm{B}-\mathrm{R}}\right)^{+} .
$$

\section{ReActive JAMMing}

In reactive jamming, the jammers only jam when detecting that the legitimate nodes are transmitting. When the legitimate nodes stop transmitting, they also stop jamming. However, it takes a short period of time for them to detect a transmission. So in this period, the legitimate receivers can receive and decode their needed information signals in a jamming-free way. In this paper, we assume that this period is equal to one time slot [5].

\subsection{Conventional Scheme}

In the first time slot of the conventional scheme, the legitimate and relaying nodes enjoy a jamming-free slot. However, from the second slot, they are continuously jammed. Therefore, if we consider a very large time scale, the affect of the first slot is insignificant. Note that the time scale we mention here is not related to the coherence time since the working of the conventional scheme does not depend on the coherence time as long as it is at least two time slots. As a result, we almost can approximate the secrecy rate of the conventional scheme in reactive jamming mode to that in constant jamming mode.

\subsection{Proposed Scheme}

The proposed scheme is designed as follows. In the first slot of the first phase, A and B simultaneously transmit $x_{\mathrm{A}}^{1}$ and $x_{\mathrm{B}}^{1}$, respectively. $\mathrm{R}$ receives the mixed information signal jamming-free. In the second slot,
A and B repeat their transmissions in the first slot. Since this slot is jammed, $\mathrm{R}$ use the mixed information signal estimated in the first slot to cancel its contribution in the received signal in the second slot and estimate the jamming component. In slot $i, 3 \leq i \leq l$, $\mathrm{A}$ and $\mathrm{B}$ transmit information signals $x_{\mathrm{A}}^{i-1}$ and $x_{\mathrm{B}}^{i-1}$, respectively. $\mathrm{R}$ easily removes the jamming component. The second phase is conducted in a similar way and finally $l-1$ pairs of information signals are delivered to the receivers. Obviously, the secrecy sum-rate of this case is the same as in constant jamming. Consequently, in section Numerical Results we do not distinguish these two jamming modes for both conventional and proposed schemes.

\section{Numerical Results}

In this section, we present several numerical results to show the superiority of the proposed scheme to the conventional scheme in many scenarios. In the first scenario, we consider three antennas at all nodes including three jammers. The powers at a non-jamming node $(\mathrm{A}, \mathrm{B}$, and $\mathrm{R})$ and a jamming node are 1 and $0.02^{\dagger}$, respectively. All A-R, B-R, $\mathrm{J}_{k}-\mathrm{A}, \mathrm{J}_{k}-\mathrm{B}$, and $\mathrm{J}_{k}-\mathrm{R}$ channels are circular complex Gaussian with mean of 0 and variance of 1 . Since in this paper, we focus on the estimation and removal of the jamming signals rather than on optimization of precoding vectors, we choose these precoding vectors as corresponding vectors 1 .

Figure 2 shows the maximum achievable sum-rate of the proposed and conventional schemes as the SNR is varied with different coherence times of $l$ time slots. Note that the performance of the conventional does not depend on the coherence time as A-R and B-R channels

\footnotetext{
${ }^{\dagger}$ We choose a low jamming power in order to show relative comparison between the proposed and conventional schemes. As the performance of the proposed scheme does not depend on the jamming power since all jamming signals are estimated and removed. The factors to deteriorate its performance is double noises as shown in (24) and (25).
} 


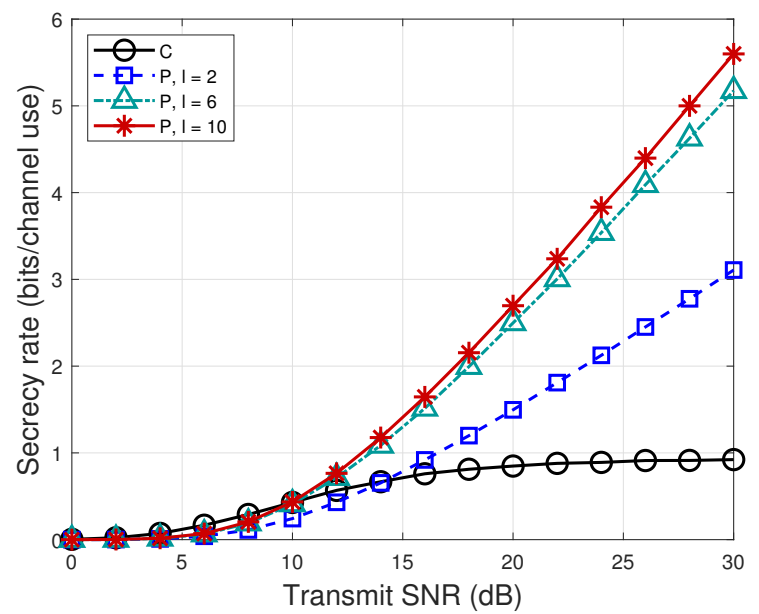

Figure 2. The proposed scheme $(\mathrm{P})$ in different coherence times, $(l$ time slots). The performance of the conventional scheme (C) does not depend on $l$.

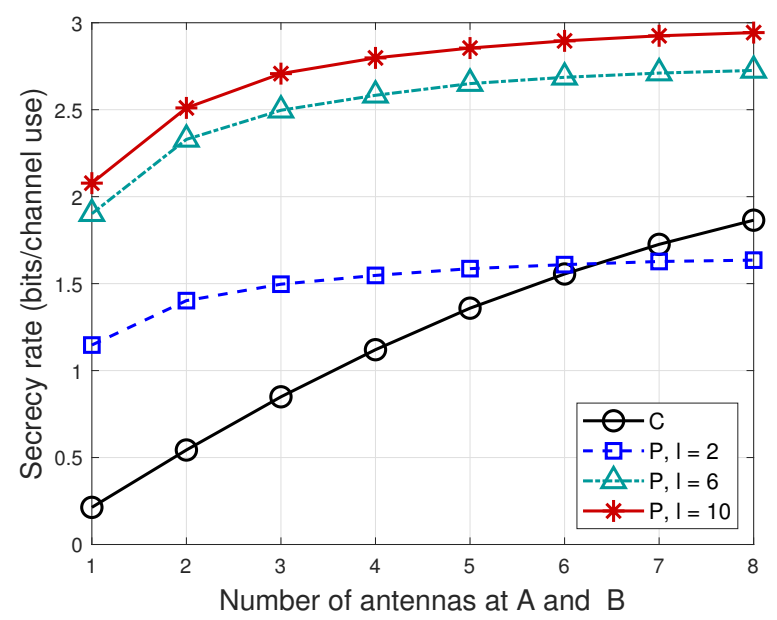

Figure 3. The effect of the number of antennas at $\mathrm{A}$ and $\mathrm{B}$ on the secrecy rate at $\mathrm{SNR}=15 \mathrm{~dB}$.

in both time slots of the scheme are perfectly known at $\mathrm{A}, \mathrm{B}$, and $\mathrm{R}$ even though they are different between two time slots. In the proposed scheme, A, B, and R sacrifice one time slot in each coherence time to estimate the jamming components which change every coherence time. At higher SNR regime, the proposed scheme is better and surpass the conventional scheme more and more since the double noises get less effective. In the meantime, it also improves with the coherence time since with a long coherence time the sacrificed time slot becomes insignificant.

Figure 3 shows the effect of the number of antennas at $\mathrm{A}$ and $\mathrm{B}$ on the secrecy rate at SNR of $15 \mathrm{~dB}$. The performance of the conventional scheme increases faster than that of the proposed scheme since the former is affected by the interference (jamming signals) whose effect can be reduced by a larger number of receiving antennas while the latter is affected by the noise whose effect can be increased. When the number of antennas at $\mathrm{R}$ is increased as shown in Figure 4, the performance of the proposed scheme also increases but not rapidly. The

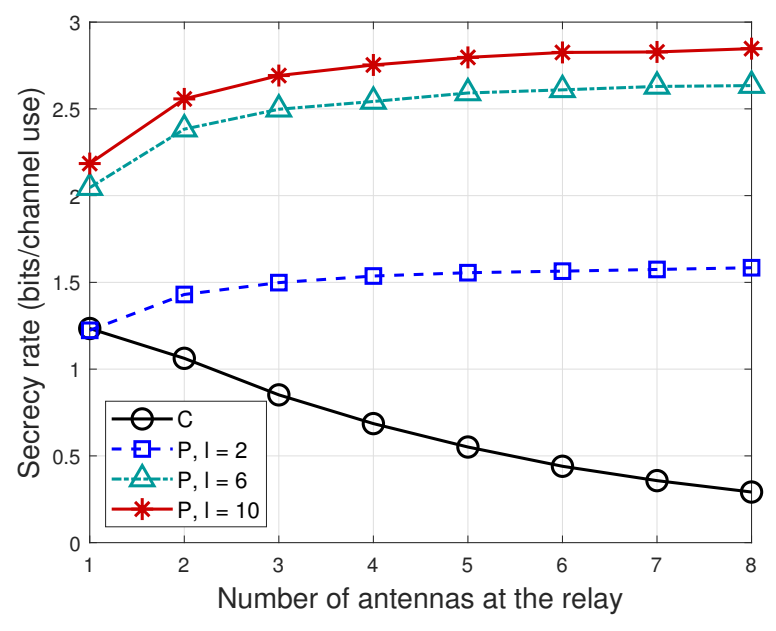

Figure 4. The effect of the number of antennas at the relay on the secrecy rate at $\mathrm{SNR}=15 \mathrm{~dB}$.

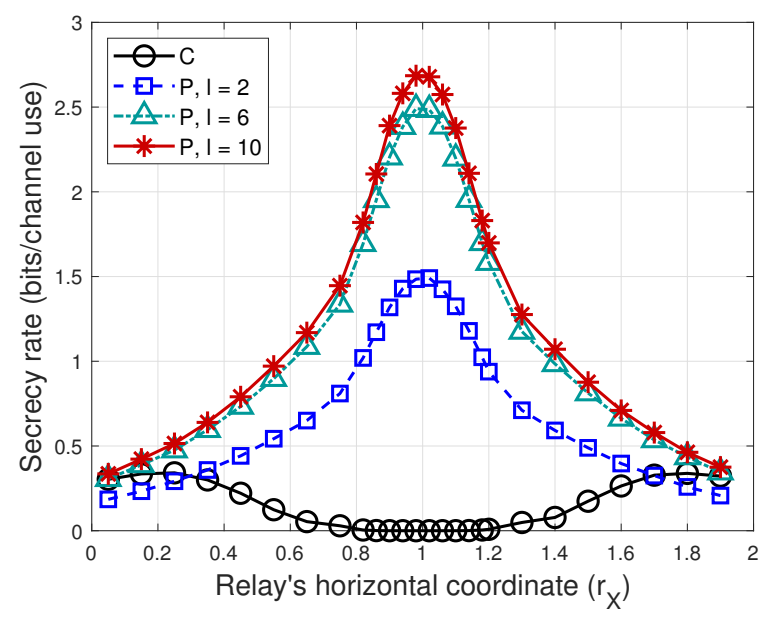

Figure 5. The affect of the relay's horizontal coordinate, $r_{\mathrm{X}}$, on the secrecy rate.

relay can reduce the affect of the jamming signals more effectively, however, at the same time, it also increases its decoding rate therefore the secrecy rate is finally decreased since the leaked rate is larger.

To analyze the affect of the positions of the nodes to the performance of the schemes, we consider the scenario where the all channel gains are given by $d^{-\frac{3}{2}} h$ in which $d$ is the physical distance between the considered transmitter and receiver, 3 is the power path loss coefficient in the non-line of sight wireless module, and $h$ is the circular complex Gaussian random variable with 0 mean and 1 variance (as described in Section 2). $A, J_{k}, R$, and $B$ are respectively located at $(0,1)$, $(1,1),\left(r_{X}, 1\right)$, and $(2,1)$ positions. The SNR is fixed at $15 \mathrm{~dB}$. The affect of the relay's horizontal coordinate on the secrecy rate is shown in Figure 5. As with other two-way relay scenarios, the performance of the proposed scheme maximizes at the middle since all jamming signals are removed. However, as they are not removed in the conventional scheme, its performance minimizes here. 


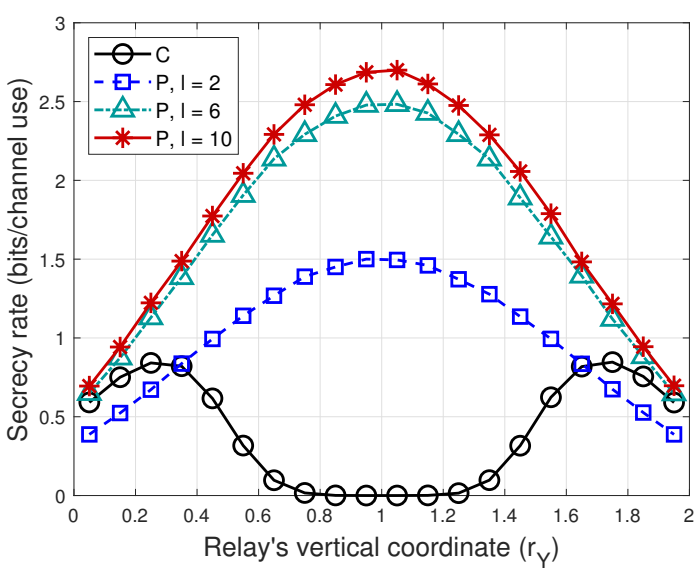

Figure 6. The affect of the relay's vertical coordinate, $r_{Y}$, on the secrecy rate.

$\mathrm{A}, \mathrm{J}_{k}$, and $\mathrm{B}$ are kept at the same positions as in Figure 5. However, the relay $\left(1, r_{Y}\right)$ moves from (1, $0)$ to $(1,2)$, i.e., on the line perpendicular to the line connection $\mathrm{A}$ and $\mathrm{B}$ positions. This means that the affect of the position change is weaker therefore the the curve near the maxima are more rounded than in the previous case. We can find the maxima of the performance of the conventional scheme near $r_{Y}=0.3$ and $r_{Y}=1.7$. These two maximum values are symmetrical across the line of $r_{Y}=1$. At these maxima, there is a balance between the negative affect of the jammers and the positive affect of the optimal position of the relay which both culminate at $r_{Y}=1$.

\section{Conclusion}

In this paper, we have considered a two-way relay network with multi-antenna legitimate, relaying, and jamming nodes. We proposed a novel scheme to estimate and eliminate the jamming signals in case of constant and reactive jamming modes. The numerical results showed that the secrecy rate of the proposed scheme is larger than that of the conventional scheme. The superiority of the proposed scheme increases with the coherence time. Generally, the performance of the conventional scheme is affected by interference and therefore significantly improved by a larger number of antennas at A and B. Meanwhile, that of the proposed scheme is affected by doubled and amplified noise due to the process of eliminating jamming signals and therefore slightly improved by a larger number of antennas at $\mathrm{A}$ and $\mathrm{B}$. The larger number of antennas at the relay helps to reduce the effect of the jamming but also increases the leaked rate. The optimal position of the relay is achieved at the midpoint between A and B as in other relay networks.

\section{ACKNOWLEDGMENT}

This study was funded by Scientific Research Project B2019-VGU-05, Ministry of Education and Training (MOET) of Vietnam.

\section{REFERENCES}

[1] A. Mukherjee, S. Fakoorian, J. Huang, and A. Swindlehurst, "Principles of physical layer security in multiuser wireless networks: A survey," IEEE Communications Surveys $\mathcal{E}$ Tutorials, vol. 16, no. 3, pp. 1550-1573, Feb. 2014.

[2] Y. Wu, A. Khisti, C. Xiao, G. Caire, K. Wong, and X. Gao, "A survey of physical layer security techniques for $5 \mathrm{G}$ wireless networks and challenges ahead," IEEE Journal on Selected Areas in Communications, vol. 36, no. 4, pp. 679-695, Apr. 2018.

[3] T. Lv, H. Gao, and S. Yang, "Secrecy transmit beamforming for heterogeneous networks," IEEE Journal on Selected Areas in Communications, vol. 33, no. 6, pp. 1154-1170, Jun. 2015.

[4] J. Y. Ryu, J. Lee, and T. Q. S. Quek, "Confidential cooperative communication with trust degree of potential eavesdroppers," IEEE Transactions on Wireless Communications, vol. 15, no. 6, pp. 3823-3836, 2016.

[5] Q. Yan, H. Zeng, T. Jiang, M. Li, W. Lou, and Y. T. Hou, "Jamming resilient communication using MIMO interference cancellation," IEEE Transactions on Information Forensics and Security, vol. 11, no. 7, pp. 1486-1499, Jul. 2016.

[6] M. Karlsson, E. Björnson, and E. G. Larsson, "Jamming a TDD point-to-point link using reciprocity-based MIMO," IEEE Transactions on Information Forensics and Security, vol. 12, no. 12, pp. 2957-2970, 2017.

[7] A. Mukherjee and A. L. Swindlehurst, "Jamming games in the mimo wiretap channel with an active eavesdropper," IEEE Transactions on Signal Processing, vol. 61, no. 1, pp. 82-91, 2013.

[8] L. Xiao, X. Lu, D. Xu, Y. Tang, L. Wang, and W. Zhuang, "UAV relay in VANETs against smart jamming with reinforcement learning," IEEE Transactions on Vehicular Technology, vol. 67, no. 5, pp. 4087-4097, 2018.

[9] C. Pu, "Jamming-resilient multipath routing protocol for flying Ad Hoc networks," IEEE Access, vol. 6, pp. 68472 68 486, 2018.

[10] L. Xiao, T. Chen, J. Liu, and H. Dai, "Anti-jamming transmission stackelberg game with observation errors," IEEE Communications Letters, vol. 19, no. 6, pp. 949-952, 2015.

[11] L. Li, A. P. Petropulu, and Z. Chen, "MIMO secret communications against an active eavesdropper," IEEE Transactions on Information Forensics and Security, vol. 12, no. 10, pp. 2387-2401, Oct. 2017.

[12] S. Vahidian, S. Hatamnia, and B. Champagne, "On the security analysis of a cooperative incremental relaying protocol in the presence of an active eavesdropper," IEEE Access, vol. 7, pp. $181812-181$ 828, Sep. 2019.

[13] T. Lv, Y. Yin, Y. Lu, S. Yang, E. Liu, and G. Clapworthy, "Physical detection of misbehavior in relay systems with unreliable channel state information," IEEE Journal on Selected Areas in Communications, vol. 36, no. 7, pp. 15171530, Jul. 2018.

[14] H. Pirayesh, P. K. Sangdeh, and H. Zeng, "Securing ZigBee communications against constant jamming attack using neural network," IEEE Internet of Things Journal, vol. 8, no. 6, pp. 4957 - 4968, 2021.

[15] K. Panyim, T. Hayajneh, P. Krishnamurthy, and D. Tipper, "On limited-range strategic/random jamming attacks in wireless ad hoc networks," in Proceedings of the IEEE 34th Conference on Local Computer Networks, 2009, pp. 922-929.

[16] M. Spuhler, D. Giustiniano, V. Lenders, M. Wilhelm, and J. B. Schmitt, "Detection of reactive jamming in DSSSbased wireless communications," IEEE Transactions on Wireless Communications, vol. 13, no. 3, pp. 1593-1603, 2014.

[17] C. Zhang, J. Ge, J. Li, F. Gong, and H. Ding, “Complexityaware relay selection for $5 \mathrm{G}$ large-scale secure two-way relay systems," IEEE Transactions on Vehicular Technology, 
vol. 66, no. 6, pp. 5461-5465, 2017.

[18] D. Tse and P. Viswanath, "Fundamentals of wireless communications," Cambridge Univ. Pr., 2005.

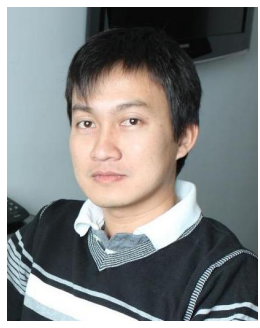

Chan Dai Truyen Thai received the B.S. degree from Posts and Telecommunications Institute of Technology (PTIT), Ho Chi Minh City, Vietnam; the M.Sc. degree from Korea Advanced Institute of Science and Technology (KAIST), Daejeon, South Korea; and the Ph.D. degree from Aalborg University, Denmark, in 2003, 2008, and 2012, respectively. He was with IFSTTAR, LEOST, Villeneuve d'Ascq, France; with Singapore University of Technology and Design (SUTD); and is now the Academic Coordinator cum Senior Lecturer of the Electrical and Computer Engineering (ECE) Study Program, Vietnamese-German University (VGU). His research interests include cooperative communications, vehicle-to-vehicle communications, communication for high-speed vehicles, security in wireless communications, and security in smart grid.

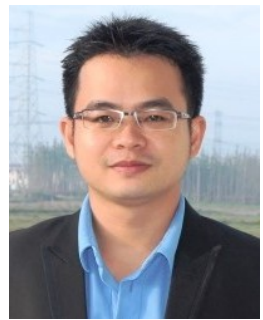

De-Thu Huynh received the Ph.D. degree in Computer Science from Huazhong University of Science and Technology, China in 2015. He is working as a lecturer and researcher in the Faculty of Information Technology at Ho Chi Minh City University of Economics and Finance, Vietnam. His current research interests were in the areas of Wireless Sensor Networks, Wireless Body Area Networks, Device-to-Device Communications, Internet of Things, and Network Security.

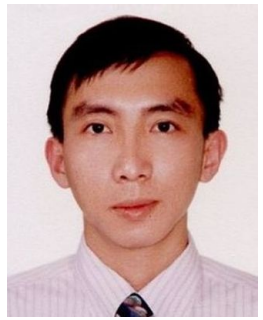

Vo Nguyen Quoc Bao (SMIEEE) is an associate professor of Wireless Communications at Posts and Telecommunications Institute of Technology (PTIT), Vietnam. He is currently serving as the Dean of Faculty of Telecommunications and the Director of the Wireless Communication Laboratory (WCOMM). His research interests include wireless communications and information theory with current emphasis on MIMO systems, cooperative and cognitive communications, physical layer security, and energy harvesting. He is the Technical Editor in Chief of REV Journal on Electronics and Communications. He is also serving as an Associate Editor of EURASIP Journal on Wireless Communications and Networking, an Editor of Transactions on Emerging Telecommunications Technologies (Wiley ETT), and VNU Journal of Computer Science and Communication Engineering. He served as a Technical Program co-chair for ATC $(2013,2014,2018)$, NAFOSTEDNICS (2014, 2015, 2016), REV-ECIT (2015, 2017), ComManTel (2014, 2015), and SigComTel $(2017,2018)$. He is a Member of the Executive Board of the Radio-Electronics Association of Vietnam (REV) and the Electronics Information and Communications Association Ho Chi Minh City (EIC). He is currently serving as vice chair of the Vietnam National Foundation for Science and Technology Development (NAFOSTED) scientific Committee in Information Technology and Computer Science (2017-2019). 